\title{
Analytical solution for postbuckling of uniform nonlinear masonry piers
}

\author{
I. Mura \\ Department of Structural Engineering, \\ University of Cagliari, Cagliari, Italy
}

\begin{abstract}
In this paper the stability condition of uniform cantilevered masonry piers, subjected to eccentric concentrated load, is investigated, considering materials with nonlinear (parabolic) stress-strain law under compression. The analytical solution is obtained by integrating the nonlinear differential equation of the deflection curve using the Taylor series method. Depending on load intensity, on the height-to-depth ratio and initial eccentricity of the applied load, a column can fail owing to elastic instability or because the masonry at the fixed section has attained, or exceeded, the allowable compressive or tensile stress.
\end{abstract}

Keywords: masonry, piers, instability, nonlinear constitutive law, Taylor series.

\section{Introduction}

The stability study of panels and columns under eccentric compression requires an accurate knowledge of the material's constitutive law, which is generally nonlinear. The experimental results reported in the literature (Powell and Hodgkinson [1], Priestley and Elder [2], Naraine and Sinha [3], Pume [4], Mojsilović [5]) and the various deformation curves that have thus far been adopted in theoretical studies of instability (Frish-Fay [7], Sawko and Rouf [8], La Mendola and Papia [9], Mura [10,11]) differ to a certain extent.

One model realistically describing the behaviour for concrete is given in a standardised material law in Eurocode2 [6]. This law simulates two behaviour limits for the material: elastic and rigid-plastic. Schematising the constitutive law with a second-degree parabolic trend, where the vertex corresponds to the maximum strength value, appears to be the most generalized approach. Indeed this schematization describes the behaviour of brick and concrete walls $[1,2]$ and will thus be used in the present study. 
As a preliminary, the stress and strain distribution are derived for a rectangular uncracked section, assuming a nonlinear (parabolic) stress-strain law under compression and that the plane section remains plane after deformation. The curvature is then derived in closed form. Pier equilibrium is then formulated in the deformed state, taking into account the effect of deflections on bearing capacity.

The analytical solution is obtained by integrating the nonlinear differential equation of the deflection curve by means of the Taylor series method (see Agnew [12] ).

\section{Symbols}

The symbols used have the following meaning:

$b \quad$ width of masonry wall or pier cross section;

$t \quad$ thickness of masonry wall or pier cross section;

$L \quad$ height of masonry wall;

$e_{0} \quad$ initial eccentricity of axial load;

$e_{I I} \quad$ added second-order eccentricity of axial load;

$e \quad$ overall load eccentricity $\left(e=e_{0}+e_{I I}\right)$;

$N \quad$ axial action;

$N_{0} \quad$ axial failure with centred load $\left(N_{0}=\sigma_{\mathrm{m}} b t\right)$;

A,B parameters characterizing stress-strain state in a section under predetermined load conditions;

$\varepsilon \quad$ longitudinal strain produced by normal stress;

$\varepsilon_{m} \quad$ maximum allowable longitudinal strain under normal stress;

$\sigma \quad$ normal compression stress;

$\sigma_{m} \quad$ maximum allowable normal compressive stress;

$\eta \quad$ transversal deflection of masonry pier at the top;

$\lambda \quad$ slenderness of masonry pier or panel $(\lambda=2 L / t)$.

\section{Theoretical premises}

For the material under compression the curve $\sigma=\sigma(\varepsilon)$ represented in figure 1(a) of the dimensionless equation is adopted:

$$
\frac{\sigma}{\varepsilon_{m}}=\frac{2 \cdot \varepsilon}{\varepsilon_{m}}-\left(\frac{\varepsilon}{\varepsilon_{m}}\right)^{2}
$$

neglecting the material's tensile strength. The latter assumption is clearly conservative for the purpose of safety evaluation. This is amply justified by experimental evidence, which shows tensile strength of non-reinforced masonry to actually be quite small compared to compression strength and by semianalytical studies [7] on the phenomenon of elastic instability of non-reinforced masonry, which have shown critical load to be little influenced by tensile strength. 
Experimental evidence has shown that once the peak of coordinates $\sigma_{m}$ and $\varepsilon_{m}$ has been attained, the curve begins to descend. The softening behaviour, not experimentally determined with any certainty, will be neglected in this study since it is not essential for determining loads of elastic instability where relatively small stresses occur.

(a)

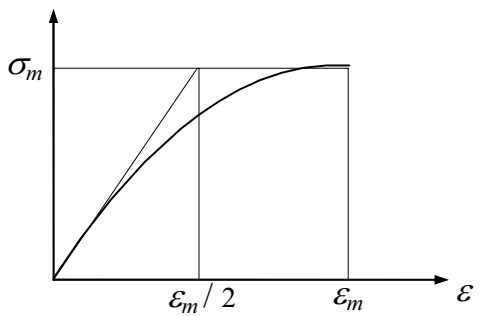

(b)

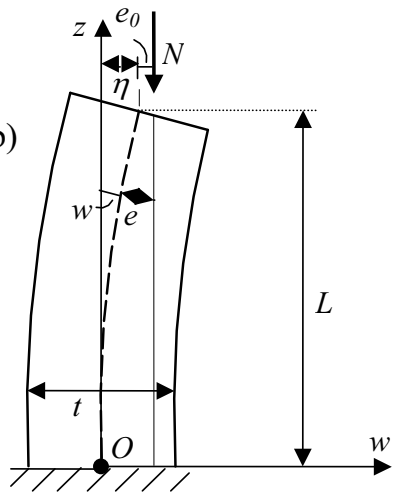

Figure 1: (a) Constitutive law of the masonry; (b) Fixed end column with vertical load $N$.

Figure 1(b) shows the structure studied, consisting of a pier subjected to eccentric compression, fixed at the base and free at the top, having a rectangular section of width $b$ and thickness $t$. Geometric eccentricity $e_{0}$ (the sum of intended and accidental eccentricities resulting) is assumed constant along the structure's axis.

\section{Stress-strain state for the section}

To determine the distribution of stress $\sigma$ and strain $\varepsilon$ through a section, we consider a pier element of infinitesimal height $\mathrm{d} z$ and dimensions $b$ and $t$ subjected to a load $N$ acting with eccentricity $e$ in figure 2 .
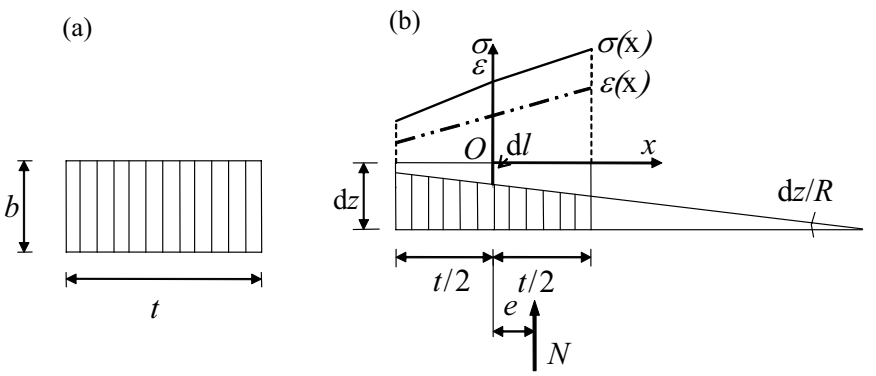

Figure 2: $\quad$ Stress and strain distribution in uncracked section. 
Since the principle of conservation of plane sections is admitted, by imposing equilibrium conditions with vertical translation and rotation, we can write the system of nonlinear equations that rigorously solves the problem [10]. Let us consider the reference system $O(x, \varepsilon)$ with origin at barycentre of the section. The following functions describe the stress and strain behaviour:

$$
\begin{aligned}
& \varepsilon(x)=\varepsilon_{m}(1-(A \cdot x+B)) \\
& \sigma(x)=\sigma_{m}\left(1-(A \cdot x+B)^{2}\right)
\end{aligned}
$$

Curvature $1 / R$ and axial shortening $\mathrm{d} l$ of the pier element are given respectively by the expressions:

$$
\begin{gathered}
\frac{1}{R}=\frac{\varepsilon(t / 2)-\varepsilon(-t / 2)}{t}=-A \cdot \varepsilon_{m} \\
\mathrm{~d} l=\varepsilon_{m}(1-B) \cdot d z
\end{gathered}
$$

The parameters $A$ and $B$ are given by:

$$
\begin{aligned}
& A=\frac{\sqrt{3}}{t}\left[\sqrt{1-\frac{N}{N_{0}}-2 \sqrt{3} \frac{N}{N_{0}} \frac{e}{t}}-\sqrt{1-\frac{N}{N_{0}}+2 \sqrt{3} \frac{N}{N_{0}} \frac{e}{t}}\right] \\
& B=\frac{1}{2}\left[\sqrt{1-\frac{N}{N_{0}}-2 \sqrt{3} \frac{N}{N_{0}} \frac{e}{t}}+\sqrt{1-\frac{N}{N_{0}}+2 \sqrt{3} \frac{N}{N_{0}} \frac{e}{t}}\right]
\end{aligned}
$$

For each assigned pair of values of dimensionless axial load $N / N_{0}$ and load eccentricity $e / t$ (so as to respect the non-cracking conditions of the section that will be given later and to avoid exceeding allowable tension), we can calculate parameters $A$ and $B$ using Eqs (6) and (7), which when introduced into Eqs (2), (3), (4) and (5) fully describe the stress-strain state of the material.

\section{Crack and failure conditions in the masonry section}

Crack conditions of the section for materials with a nonlinear elastic constitutive law depend on both eccentricity $e$ of load $N$ (as is the case with materials having linear behaviour) and on load intensity. Condition $\varepsilon(-t / 2)=0$, expressed by means of equation (2), defines the crack initiation condition. Taking into account equations (6) and (7), which give $A$ and $B$, after appropriate simplifications we arrive at a nondimensional second-degree equation that yields the limit load $\left(N / N_{0}\right)_{f}$ as a function of eccentricity $e / t$. After discarding the trivial solution, we get:

$$
\left(\frac{N}{N_{0}}\right)_{f}=\frac{2(1-6 \cdot e / t)}{3(1-4 \cdot e / t)}
$$


Regarding failure conditions, let us consider separately the two cases of an uncracked and cracked section. Referring to equation (3), obviously we must set: $\sigma(t / 2)=\sigma_{m}$. Taking into account equations (6) and (7), after appropriate simplifications we arrive at:

$$
\left(\frac{N}{N_{0}}\right)_{c}=\frac{1}{1+4 \cdot e / t}
$$

For the cracked section it has been shown [10] that the crushing failure load of the material is correlated with eccentricity by means of:

$$
\left(\frac{N}{N_{0}}\right)_{c}=\frac{16}{9} \cdot\left(\frac{1}{2}-\frac{e_{0}}{t}\right)
$$

\section{Analytical method}

Let us consider the masonry pier fixed as in figure 1(b). For studying the stability of similar slender unidimensional elements we need to take into account two distinct kinds of nonlinearity. On the one hand mechanical nonlinearity, originating from the material's constitutive law, on the other, geometric nonlinearity derived from second-order effects (increases in load eccentricity due to element deflection). The differential equation for the deflection curve is given by:

$$
w^{\prime \prime}=\frac{N \cdot e}{R}
$$

where $R$ is flexural rigidity, which varies along axis $z$ as eccentricity $e$ varies. For each pier section the findings of the author's earlier studies hold true, so long as eccentricity $e / t$ is substituted with $\left(e_{0}+e_{I I}\right) / t$ where, as we can see in figure 1(b), we have $e_{I I}=\eta-w$.

The differential equation (10) of the deflection curve is rewritten as follows:

$$
w^{\prime \prime}=\frac{\sqrt{3} \varepsilon_{m}}{t}\left[\sqrt{1-\frac{N}{N_{0}}\left(1+2 \sqrt{3}\left(\frac{e_{0}}{t}+\frac{\eta}{t}-\frac{w}{t}\right)\right)}-\sqrt{1-\frac{N}{N_{0}}\left(1-2 \sqrt{3}\left(\frac{e_{0}}{t}+\frac{\eta}{t}-\frac{w}{t}\right)\right)}\right]
$$

\subsection{Buckling calculation for centred load}

For centred load $\left(e_{0} / t=0\right)$ if we simply want to determine the numerical value of the buckling load, then we observe that under critical equilibrium conditions the inflected configurations are infinitely close to the straight ones. If we consider the quantity $(\eta-w) / t$ in equation (11) to be infinitesimal, we can expand 
the roots on the right hand side in the Taylor's series. Truncating at the second term yields, after rearrangement:

$$
w^{\prime \prime}+\alpha^{2} \cdot w=\alpha^{2} \cdot \eta
$$

where:

$$
\alpha^{2}=\frac{\frac{6 \cdot \varepsilon_{m} \cdot N}{t \cdot N_{0}}}{\sqrt{1-\frac{N}{N_{0}}}}
$$

Studying the above differential equation (12) does not present any difficulties. We arrive at the well-known expression:

$$
\alpha \cdot L=\frac{\pi}{2}+k \cdot \pi
$$

obtaining the smallest value of the critical load for $k=0$. From equation (14) we deduce:

$$
\left(\frac{N}{N_{0}}\right)_{c r i t}=\frac{1}{2} \cdot \frac{\pi^{4}}{36 \cdot \varepsilon_{m}^{2} \cdot \lambda^{4}}\left(\sqrt{1+4 \cdot \frac{36 \cdot \varepsilon_{m}^{2} \cdot \lambda^{4}}{\pi^{4}}}-1\right)
$$

Equation (15) coincides with the well-known Engesser critical load formula (the tangent-modulus theory of buckling load) for the column built with material having nonlinear elastic behaviour whose constitutive law is expressed by equation (1).

\subsection{Integration by Taylor series method}

The differential equation (11) is a classic second order non-linear Newton equation of the type $w^{\prime \prime}=g(w)$. Finding the general integral in closed form proves extremely difficult because of the complexity of the functions contained in the second term. Approximate solutions can be obtained by means of the integration method, which uses Taylor series expansion.

Considering the origin of the reference system $O$ as the starting point for the expansion $(z=0)$, we get:

$$
w(z)=w(0)+w^{\prime}(0) \cdot z+w^{\prime \prime}(0) \cdot \frac{z^{2}}{2 !}+w^{\prime \prime \prime}(0) \cdot \frac{z^{3}}{3 !}+\ldots \ldots
$$

The first two coefficients can be immediately deduced, taking into account that at the origin the deflection and the tangent should be zero. We set:

$$
\begin{aligned}
& w(0)=0 \\
& w^{\prime}(0)=0
\end{aligned}
$$


Taking into account equations (17) and (18), it is easy to deduce the third coefficient of the Taylor series expansion from equation (16). Assuming:

$$
\begin{aligned}
& K_{1}=\left[1-\frac{N}{N_{0}}-2 \sqrt{3} \cdot \frac{N}{N_{0}}\left(\frac{e_{0}}{t}+\frac{\eta}{t}\right)\right] \\
& K_{2}=\left[1-\frac{N}{N_{0}}+2 \sqrt{3} \cdot \frac{N}{N_{0}}\left(\frac{e_{0}}{t}+\frac{\eta}{t}\right)\right]
\end{aligned}
$$

we obtain:

$$
w^{\prime \prime}(0)=-\frac{\sqrt{3} \cdot \varepsilon_{m}}{t}\left(\sqrt{K_{1}}-\sqrt{K_{2}}\right)
$$

The fourth and subsequent coefficients can be obtained further deriving from equation (16) with respect to the variable $z$ and considering that after each derivation the values of the coefficients previously calculated can be substituted into the right hand side. Thus we get:

$$
\begin{aligned}
w^{\prime \prime \prime}(0) & =0 \\
w^{i v}(0) & =\frac{1}{2 t}\left(\frac{-\sqrt{3} \varepsilon_{m}}{t}\right)^{2}\left(2 \sqrt{3} \frac{N}{N_{0}}\right)\left[\frac{K_{1}-K_{2}}{\sqrt{K_{1} \cdot K_{2}}}\right] \\
w^{v}(0) & =0 \\
w^{v i}(0) & =\frac{1}{4 t^{2}}\left(\frac{-\sqrt{3} \varepsilon_{m}}{t}\right)^{3}\left(2 \sqrt{3} \frac{N}{N_{0}}\right)^{2} \frac{1}{K_{1} \cdot K_{2}}\left(\sqrt{K_{1}}-\sqrt{K_{2}}\right)\left(\sqrt{K_{1}}+\sqrt{K_{2}}\right)^{2} \\
& +\frac{3}{4 t^{2}}\left(\frac{-\sqrt{3} \varepsilon_{m}}{t}\right)^{3}\left(2 \sqrt{3} \frac{N}{N_{0}}\right)^{2}\left(K_{1}+K_{2}\right) \frac{K_{1} \sqrt{K_{1}}-K_{2} \sqrt{K_{2}}}{K_{1} K_{2} \sqrt{K_{1}} \sqrt{K_{2}}} \\
& -\frac{6}{4 t^{2}}\left(\frac{-\sqrt{3} \varepsilon_{m}}{t}\right)^{3}\left(2 \sqrt{3} \frac{N}{N_{0}}\right)^{2} \frac{K_{1} \sqrt{K_{1}}-K_{2} \sqrt{K_{2}}}{K_{1} K_{2} \sqrt{K_{1}} \sqrt{K_{2}}}
\end{aligned}
$$

and so forth.

The load-deflection response diagram can be obtained from equation (16), introducing the coefficients calculated above. Truncating the series at the third significant term and assuming:

$$
w(z)=w^{\prime \prime}(0) \cdot \frac{z^{2}}{2}+w^{i v}(0) \cdot \frac{z^{4}}{4 !}+w^{v i}(0) \cdot \frac{z^{6}}{6 !}
$$

considering that $w(L)=\eta$ and having introduced slenderness $\lambda=2 L / t$ we get: 


$$
\begin{aligned}
& {\left[\left(\frac{\eta}{t}\right)^{2} K_{1} K_{2}+K_{3}^{2}-K_{4}^{2}\left(K_{1}+K_{2}\right) K_{1} K_{2}-K_{5}^{2}\left(K_{1}+K_{2}\right)-4 K_{4} K_{5} K_{1} K_{2}\right]^{2}} \\
& -\left[2 \frac{\eta}{t} K_{3}-2 K_{4} K_{5}\left(K_{1}+K_{2}\right)-2 K_{4}^{2} K_{1} K_{2}-2 K_{5}^{2}\right] K_{1} K_{2}=0
\end{aligned}
$$

with $K_{3}, K_{4}$ and $K_{5}$ given by the following further positions:

$$
\begin{gathered}
K_{3}=\frac{1}{48}\left(\frac{\sqrt{3} \lambda^{2} \varepsilon_{m}}{4}\right)^{2}\left(2 \sqrt{3} \frac{N}{N_{0}}\right)\left(K_{1}-K_{2}\right) \\
K_{4}=\frac{1}{1440 \cdot K_{1} K_{2}}\left[\left(\frac{\sqrt{3} \lambda \varepsilon_{m}}{4}\right)^{3}\left(2 \sqrt{3} \frac{N}{N_{0}}\right)\left(K_{1}+K_{2}\right)-720\left(\frac{\sqrt{3} \lambda \varepsilon_{m}}{4}\right) K_{1} K_{2}\right] \\
K_{5}=\frac{1}{2880 \cdot K_{1} K_{2}}\left(\frac{\sqrt{3} \lambda \varepsilon_{m}}{4}\right)^{3}\left(2 \sqrt{3} \frac{N}{N_{0}}\right)\left[3\left(K_{1}+K_{2}\right)^{2}-4 K_{1} K_{2}\right]
\end{gathered}
$$

\section{Numerical results}

We can study equation (27) for the two cases of centred load $\left(e_{0} / t=0\right)$ and load with small eccentricity $\left(e_{0} / t \neq 0\right)$.

For the centred load we can write:

$$
\left(\frac{\eta}{t}\right)^{2}\left[\left(\frac{\eta}{t}\right)^{14} C_{14}\left(\frac{N}{N_{0}}\right)+\left(\frac{\eta}{t}\right)^{12} \cdot C_{12}\left(\frac{N}{N_{0}}\right)+\ldots . .+\left(\frac{\eta}{t}\right)^{2} C_{2}\left(\frac{N}{N_{0}}\right)+C_{0}\left(\frac{N}{N_{0}}\right)\right]=0
$$

with $C_{14}, C_{12}, \ldots C_{0}$, polynomials of $N / N_{0}$.

Equation (31) (also referring to figure 3, determined for exemplary purposes assuming $\lambda=15$ e $\left.\varepsilon_{m}=.004\right)$ can be readily interpreted. The quantity not enclosed in square brackets leads to the dual solution $\eta / t=0$, which determines column straightness and identifies the (ascending) path of the stable equilibrium points. The (descending) path of the unstable equilibrium points can instead be derived from the square bracketed expression, set equal zero. This is obtained by means of a numerical procedure and is given by the curve that identifies the smallest actual values of $N / N_{0}$ for predetermined $\eta / t$.

The load at which critical equilibrium is reached is obtained as the point of intersection between the stable equilibrium and the unstable equilibrium paths.

Setting $\eta / t=0$ in the square bracketed expression in equation (31) and equating the coefficient $C_{0}$ to zero, the smallest actual value of $N / N_{0}$ is given by:

$$
\left(\frac{N}{N_{0}}\right)_{\text {crit }}=\frac{1}{2} \cdot \frac{16}{K \cdot 3 \cdot \varepsilon_{m}^{2} \cdot \lambda^{4}}\left(\sqrt{1+4 \cdot \frac{K \cdot 3 \cdot \varepsilon_{m}^{2} \cdot \lambda^{4}}{16}}-1\right)
$$


with:

$$
K=\sqrt[3]{\frac{7 \sqrt{14}}{900}+\frac{1519}{5400}}+\sqrt[3]{\frac{7 \sqrt{14}}{900}}-\frac{1519}{5400}+\frac{2}{3} \cong 1.97554461
$$

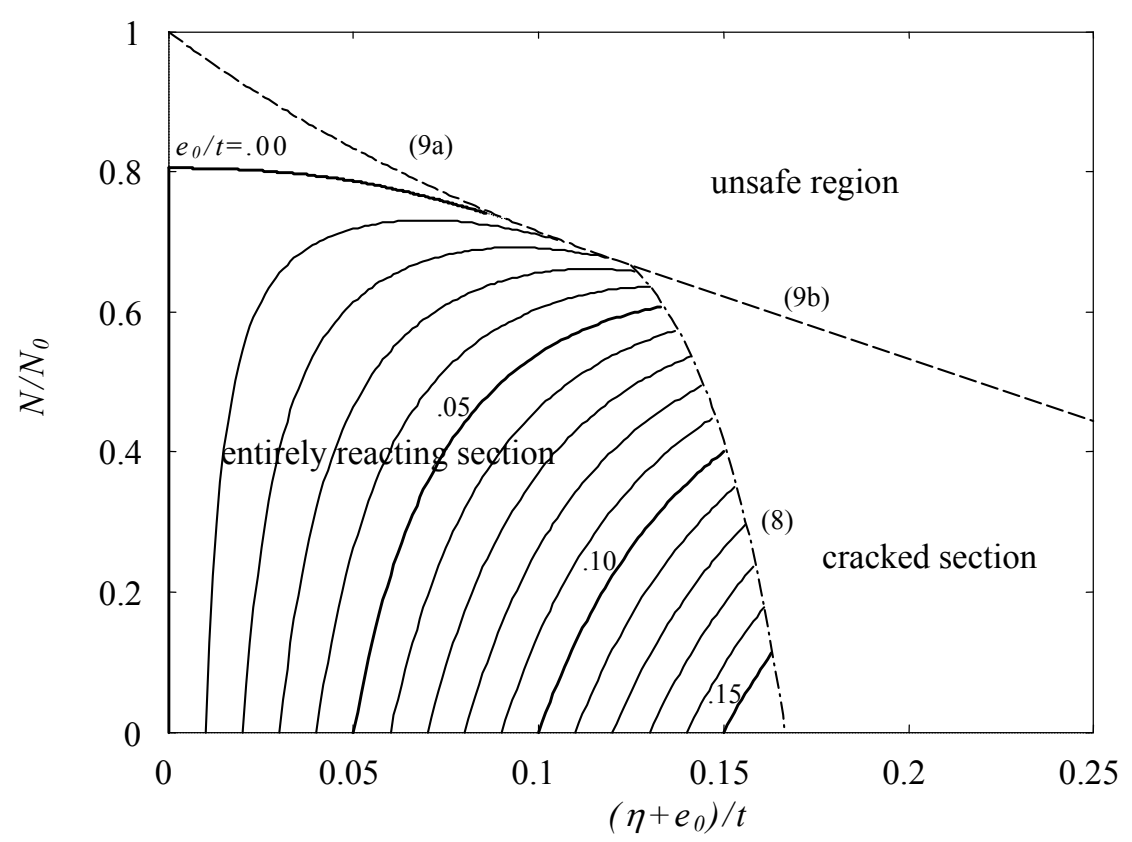

Figure 3: Load-deflection response diagrams with slenderness $\lambda=15$ for some values of dimensionless eccentricity $e_{0} / t$.

For the case of small eccentricity we used a numerical procedure for studying equation (27), obtaining curves of the type shown in figure 3 . Here we can readily identify both the ascending path of stable equilibrium, the descending path of unstable equilibrium as well as the point of critical equilibrium separating the two, which coincides with the critical load. The curves plotted in figure 3 are physically meaningful only in the safe zone for the section subjected to the greatest stress (the fixed section, at the base), which is delineated by the material's crack and crush curves. When the ascending portion of the stability curves intercepts the crack or crush curves, the limit state to be considered is precisely that at which cracking or crushing occurs.

In figure 3 the representative load shown on the $y$-axis is the nondimensionalized load $N / N_{0}$. The representative deflections shown on the $\mathrm{x}$ axis are also nondimensional $\left(\eta+e_{0}\right) / t$ and determine the sum of initial load eccentricity and deflection at the pier top. Finally, the reference condition is the unloaded pier with no deflection. 
Table 1: $\quad$ Comparison of buckling loads for some values of slenderness $\lambda$.

\begin{tabular}{|c|c|c|c|}
\hline$\lambda$ & $\begin{array}{c}\text { buckling load using } \\
\text { Taylor series } \\
(32)\end{array}$ & $\begin{array}{c}\text { buckling load using } \\
\text { Engesser solution } \\
(15)\end{array}$ & $\begin{array}{c}\text { Error \% } \\
(32)-(15)\end{array}$ \\
\hline 5 & .9963230 & .9963313 & -.00083 \\
\hline 10 & .9468646 & .9469278 & -.01143 \\
\hline 15 & .8053838 & .8056813 & -.03692 \\
\hline 20 & .6270964 & .6274827 & -.06156 \\
\hline 25 & .4758301 & .4762014 & -.07796 \\
\hline
\end{tabular}

As using three significant terms makes the variables $N / N_{0}$ e $\eta / t$ in both equation (27) and in the square bracketed expression of equation (31) very large, we determined the postbuckling curves in figure 3 numerically, using a simple automatic calculation program.

For predetermined values of $\eta / t$ we first discretized the nondimensionalized load $N / N_{0}$, identifying the steadily increasing values from zero upward. We then calculated the value of the function at those points, finding the next two load values at which the function changed sign. After obtaining the buckling load interval, the bisection method was applied thereto to accurately compute the buckling load for which the function value is zero.

Table 1 shows the comparison between instability loads for centred loads, obtained using equations (15) and (33) for typical column slenderness values. As can be observed, the differences are minimal and the approximate solution obtained by means of Taylor expansion practically coincides with the exact solution determined with the tangent-modulus theory.

\section{Conclusions}

We have analytically investigated the stability condition of uniform cantilevered masonry piers subjected to an eccentric concentrated top load, considering material with nonlinear (parabolic) stress-strain law under compression.

Depending on load intensity, height-to-depth ratio and initial eccentricity of the applied load, a column can fail owing to elastic instability or because the masonry at the fixed section has reached, or exceeded, the allowable compressive or tensile stress.

The proposed approach yields practically the same results as the exact solution and can be utilized to evaluate the safety condition of masonry bearing walls, such as those in buildings of historical or architectural interest.

\section{References}

[1] Powell, B., \& Hodgkinson, H.R., The determination of stress/strain relationship of brickwork. Proc. $4^{\text {th }}$ Int. Brick Masonry Conference, Brugge, 26-28 April, pp. 2.a.5-2.a.5-5, 1976. 
[2] Priestley, M.J.N., \& Elder, D.M., Stress-Strain Curves for Unconfined and Confined Concrete Masonry. ACI Journal, 80(3), pp. 192-201, 1983.

[3] Naraine, K., Sinha, S., Behavior of brick masonry under cyclic compressive loading. J. Struct. Engrg., ASCE, 1989; 115(6): 1432-1445.

[4] Pume, D., Stress-strain relationships of masonry made from Czech clay units produced between 1955 and 1992. Computer Methods in Structural Masonry - 4, Ed. by G.N. Pande, J. Middleton and B. Kralj, E\&FN London, 1998.

[5] Mojsilović, N., A discussion of masonry characteristics derived from compression tests. $10^{\text {th }}$ Canadian Masonry Symposium, Banff, Alberta, June 8-12, 2005.

[6] ENV 1992-1-1: Eurocode 2: Design of concrete structures - Part 1: General rules and rules for building. January 2001.

[7] Frish-Fay, R., Quasi-Analytical Method for the Analysis of a Masonry Column with a Non-Linear Stress-Strain Law. Int. J. of Masonry Construction, 2, pp. 41-46, 1981.

[8] Sawko, F., \& Rouf, M.A., On the stiffness properties of masonry. Proc. Instn. Civ. Engrs, Technical Note 397, 77(2), pp. 1-12, 1984.

[9] La Mendola, L., \& Papia, M., Stability of masonry piers under their own weight and eccentric load. Journal of Structural Engineering, ASCE, 119(6), pp. 1678-1693, 1993.

[10] Mura, I., Stability of No-Tension Masonry Walls having a Non-Linear Constitutive Law. In: B.H.V. Topping, editor. Proceedings of the Tenth International Conference on Civil, Structural and Environmental Engineering Computing, 2005, Civil-Comp Press, Stirling, United Kingdom, paper 192.

[11] Mura, I., Secant Stiffness for no-tension masonry walls having a nonlinear constitutive law. In: Brebbia CA, Torpiano A, editors. Structural Studies, Repairs and Maintenance of Heritage Architecture IX, WIT Press, Southampton (UK), 2005.

[12] Agnew, R., Differential Equations, Mc Graw-Hill Book Company Inc., New York, 1960. 